\title{
Antioxidant Activity of 50 Traditional Chinese Medicinal Materials Varies with Total Phenolics
}

\author{
Zhengyou He ${ }^{1,2^{*}}$, Minbo Lan ${ }^{1}$, Dongying Lu ${ }^{1}$, Hongli Zhao ${ }^{1}$, Huihui Yuan ${ }^{1}$ \\ ${ }^{1}$ Research Center of Analysis \& Test and Institute of Advanced Materials, East China University of Science \& Technology, \\ Shanghai, China \\ ${ }^{2}$ Sichuan Industrial Institute of Antibiotics, Chengdu University, Chengdu, China \\ Email: Hezhengyou@aliyun.com
}

Received September 15, 2013; revised November 14, 2013; accepted November 29, 2013

Copyright (C) 2013 Zhengyou He et al. This is an open access article distributed under the Creative Commons Attribution License, which permits unrestricted use, distribution, and reproduction in any medium, provided the original work is properly cited.

\begin{abstract}
This study was designed to determine the total phenolic content of 50 herbs and to examine their antioxidant potential. In the sample preparation, $60 \%$ ethanol was chosen as the extraction solvent for the subsequent experiments. Folin-Cicolteau phenol reagent and a colorimetric method were used to determine the total phenolic content of the selected herbs. The result showed that total phenolic content of those herbs ranged from 2 to $185 \mathrm{mg} / \mathrm{g}$. In antioxidant assay, the ferric reducing/antioxidant power (FRAP) values ranged from 2 to $134 \mathrm{mg} \mathrm{GAE} / \mathrm{g}$; the $I C_{50}$ values of DPPH $\bullet \cdot \cdot \mathrm{OH}$ and $\mathrm{O}_{2}^{-} \quad$ scavenging were in the range of $0.06-5.50 \mathrm{mg} / \mathrm{mL}, 0.017-0.636 \mathrm{mg} / \mathrm{mL}$ and $0.050-0.681 \mathrm{mg} / \mathrm{mL}$ respectively.

Flos caryophylli was the exceptant in the $\mathrm{O}_{2}^{--}$scavenging assay because there was no linear relation between the concentration and the scavenging percentage. Compared to gallic acid, ascorbic acid and butylated hydroxytoluene (BHT) in antioxidant assay as positive control, the most potential antioxidant herbs were Cacumen platycladi, Radix et Rhizoma rhei, Rhizoma rhodiolae crenulatae, and Rhizoma sanguisorbae with considerable content of phenolics. Especially, a positive and significant correlation was found between the total phenolic content and FRAP value or DPPH• scavenging percentage.
\end{abstract}

Keywords: Traditional Chinese Medicinal Material; Total Phenolics; Antioxidant Activity; Ferric Reducing/Antioxidant Power; Free Radical Scavenging Activity

\section{Introduction}

Roles of the reactive oxygen species (ROS) and reactive nitrogen species (RNS) are increasingly recognized in physiological processes, pathogenesis of many diseases, and molecular mechanisms in many drug-therapies [1]. ROS are generated by all aerobic organisms and their production seems to be essential for signal-transduction pathways that regulate multiple physiological processes. Excessive amount of ROS, however, can initiate toxic and lethal chain reactions, which disable the biological structures that are required for cellular integrity and survival. Recently, there is a growing interest in substances exhibiting antioxidant properties that are supplied to human and animal organisms as food components or as specific redox-therapy drugs [1]. Substantive experiments have already testified that many phytochemicals and extracts from plants possess antioxidant effects.

\footnotetext{
"Corresponding author.
}

Many synthetic antioxidants, such as butylated hydroxyanisole (BHA), butylated hydroxytoluene (BHT) and tert-butylhydro-quinone (TBHQ), are widely used in food and pharmaceutical industries against oxidative damage. However, animal tests have demonstrated that those synthesized compounds would accumulate in rats and result in liver-damage and carcinogenesis [2]. Interestingly, some important antioxidants, including ascorbic acid and the tocopherols, cannot be synthesized by humans and must be taken in diet [3]. It has long been recognized that some naturally occurring substances in plants process antioxidant activity. Therefore, the development and utilization of more effective and non-toxic antioxidants from natural products are desired, not only for the food and drug storage, but also for the nutritional and clinical applications.

It is well known that the traditional Chinese herbs have been used in food and medicine over two thousand years. There are more than 11,000 officinal plants, 1500 offici- 
nal animals and 80 officinal minerals used as the traditional Chinese medicine [4]. For the reason of biodiversity, the chemical composition and bioactivity of the medicinal materials are also varied. Epidemiological studies have shown that many natural antioxidant compounds possess anti-inflammatory, antiatherosclerotic, antitumor, antimutagenic, anticarcinogenic, antibacterial, or antiviral activities to a greater or lesser extent [5,6]. Apparently, the Chinese medicinal plants may contain a wide variety of chemical composition, including phenolic compounds (e.g. phenolic acids, flavonoids, quinones, coumarins, lignans, stilbenes, tannins), nitrogen compounds (alkaloids, amines, betalains), vitamins, terpenoids (including carotenoids), with potential antioxidant activities [7]. In free radical biology, the balance between antioxidation and oxidation is believed to be a critical concept to maintain a healthy biological system, which is similar to the concept of the balance between "Yin" and "Yang" in the Traditional Chinese Medicine (TCM). The effective compositions in the yin-tonic herbs were mainly flavonoids with strong antioxidant activities six times higher than that of the yang-tonic herbs [8]. Contrarily, Szeto and Benzie indicated that the yin nature of herbs may not be necessarily associated with superior antioxidative effect to yang-tonic herbs, at least in terms of DNA protection against oxidant challenge [9]. The synergetic antioxidant effects of the traditional Chinese herbs should be considered in the view of systems biology [10], but the literature partially revealed the inner correlation between the antioxidant capacity and the traditional usages. Consequently, it is necessary to evaluate the antioxidant activity of traditional Chinese herbs systematically using different types of free radical.

50 Traditional Chinese herbs, grown and processed under the standard operating procedures, were selected and prepared for the initial investigation. According to the classification of their traditional usages [4], 18 of those herbs, including 11 stanchers, are used as the haematic. The next is the heat-clearing drug, and 10 medicinal materials are ranged to this class. The third is the tonic, including one yin-tonic, eight yang-tonics, and four weaktonics. Other medicinal materials are sorted into diaphoretic, damp-resolving, cathartic, and antitussive respectively. The main objectives of this paper were a) to determine the content of total phenolics in above medicinal materials; b) to evaluate their in vitro antioxidant activity of ferric reducing and antioxidant power (FRAP), and free radicals $\left(\mathrm{DPPH} \bullet, \bullet \mathrm{OH}\right.$ and $\mathrm{O}_{2}^{--}$) scavenging capacities.

\section{Materials and Methods}

\subsection{Materials from the Traditional Chinese Medicine}

50 Chinese medicinal materials were purchased from a local pharmacy (Jiamei medicine chain Co., Ltd., Shanghai, China). The planting, harvesting, drying, processing, and storage of the medicinal materials were conducted according to strict traditional procedures, namely the standard operating procedures implemented in China. Names of those Chinese medicinal materials are listed in Table 1, all of them have been identified according to the literature [4]. All the voucher specimens have been deposited at the Specimen-room of the Research Center of Analysis \& Test, East China University of Science and Technology, Shanghai, China.

\subsection{Preparation of Extracts}

Dried and pulverized sample ( $1 \mathrm{~g}$ ) was extracted using 20 $\mathrm{ml}$ of $60 \%(\mathrm{v} / \mathrm{v})$ ethanol. It was mixed continuously with magnetic stirrer under refluxing at $60^{\circ} \mathrm{C}$ for $1 \mathrm{~h}$. Then, the extracts were filtered over Xinhua filter paper. The residue was re-extracted under the same conditions. The obtained extracts were conflated and concentrated in vacuo under $40^{\circ} \mathrm{C}$ using a rotary evaporator (ZX98-1 Rotavapor, Shanghai Organic Chemistry Institute, Shanghai, China) to yield dry extracts, which were stored at $4^{\circ} \mathrm{C}$ for further analysis.

\subsection{Total Phenolic Contents (TPCs) Analysis}

The TPCs of those extracts were analyzed using FolinCiocalteu's phenol reagent [11]. The extracts were dissolved in $60 \%(\mathrm{v} / \mathrm{v})$ ethanol at the concentrations to fit the TPC analysis. The solutions $(0.5 \mathrm{~mL})$ of different concentrations were put in a $10 \mathrm{~mL}$ volumetric flask, 4.5 $\mathrm{mL}$ of distilled water and $1.0 \mathrm{~mL}$ of Folin-Ciocalteu reagent were added, and the flask was shaken thoroughly. After $3 \mathrm{~min}, 4 \mathrm{~mL}$ of $2 \% \mathrm{Na}_{2} \mathrm{CO}_{3}$ was added, and the mixture was allowed to stand for $2 \mathrm{~h}$ with intermittent shaking. The absorbance was measured at $770 \mathrm{~nm}$ (UV2102, Unico Instruments Co., Ltd., Shanghai, China). Experiments were carried out in triplicate. The results were expressed as gallic acid equivalent per gram raw material (mg GAE/g). The same procedure was repeated

Table 1. Extraction efficiencies of various dilutions of ethanol in water on Folium artemistae argyi, Rhizoma rhodiolae crenulatae, and Cortex eucommiae.

\begin{tabular}{|c|c|c|c|c|}
\hline \multirow{2}{*}{ Species } & \multicolumn{4}{|c|}{ TPC $^{\text {a }}$ (mg GAE/g dried sample) } \\
\hline & $95 \%$ ethanol & $60 \%$ ethanol & $30 \%$ ethanol & $10 \%$ ethanol \\
\hline $\begin{array}{l}\text { Folium } \\
\text { artemistae } \\
\text { argyi }\end{array}$ & $18.49 \pm 0.46$ & $34.72 \pm 0.72$ & $28.33 \pm 0.70$ & $24.13 \pm 0.56$ \\
\hline $\begin{array}{l}\text { Rhizoma } \\
\text { rhodiolae } \\
\text { crenulatae }\end{array}$ & $93.61 \pm 1.72$ & $184.56 \pm 3.78$ & $169.02 \pm 3.95$ & $151.70 \pm 1.87$ \\
\hline $\begin{array}{c}\text { Cortex } \\
\text { eucommiae }\end{array}$ & $22.07 \pm 0.61$ & $40.04 \pm 0.80$ & $34.15 \pm 0.66$ & $31.28 \pm 0.59$ \\
\hline
\end{tabular}

${ }^{\mathrm{a}}$ Results are means $\pm \mathrm{SD}(\mathrm{n}=3)$. 
for all of the standard gallic acid solutions (0 - 10,000 $\mu \mathrm{g} / \mathrm{mL}$ ), and the standard curve was determined using the equation:

$$
\text { Absorbance }=0.0011 \times \text { gallic acid }(\mu \mathrm{g})+0.0027 \text {. }
$$

\subsection{Antioxidant Screening}

\subsubsection{Ferric Reducing and Antioxidant Power (FRAP) Assay}

The total antioxidant potential of those herbs was determined using ferric reducing and antioxidant power (FRAP) assay [12]. FRAP reagent was freshly prepared and mixed in the proportion of 10:1:1 (v:v:v) for A:B:C solutions, where $\mathrm{A}=300 \mathrm{mmol} / \mathrm{L}$ sodium acetate trihydrate in glacial acetic acid buffer $(\mathrm{pH}=3.6) ; \mathrm{B}=10$ $\mathrm{mmol} / \mathrm{L} \mathrm{TPTZ} \mathrm{in} 40 \mathrm{mmol} / \mathrm{L} \mathrm{HCl}$; and $\mathrm{C}=20 \mathrm{mmol} / \mathrm{L}$ $\mathrm{FeCl}_{3}$. Gallic acid was used for a standard curve with all solutions, including samples dissolved in $60 \%$ ethanol. The assay was carried out at $37^{\circ} \mathrm{C}(\mathrm{pH}=3.6)$ using 0.4 $\mathrm{mL}$ sample or standard solution plus $4.0 \mathrm{~mL}$ FRAP reagent shown above. After $10 \mathrm{~min}$ incubation at room temperature, the absorbance was read at $593 \mathrm{~nm}$. Results were expressed in $\mathrm{mg}$ gallic acid equivalent per gram dried herb weight (mg GAE/g). Experiments were carried out in triplicate.

\subsubsection{DPPH Radical Scavenging Activity Assay}

This spectrophotometric assay used the stable DPPH radical as the reagent to determine the $\mathrm{DPPH} \bullet$ scavenging activity [13]. The extracts and standards were dissolved in $60 \%(\mathrm{v} / \mathrm{v})$ ethanol at the concentrations to fit the DPPH assay. Ethanolic extracts or standards of 0.1 $\mathrm{mL}$ at various concentrations was added to $4.0 \mathrm{~mL} 0.004 \%$ $\mathrm{DPPH} \cdot$ methanol solution in a $10 \mathrm{~mL}$ test tube respectively. After $30 \mathrm{~min}$ incubation at room temperature, the absorbance was read against a contrast only containing all solvents at $517 \mathrm{~nm}$. Inhibition of the free radical of DPPH in percent (I\%) was calculated as follow:

$$
\text { Inhibition } \%=\left[\left(\mathrm{A}_{\text {blank }}-\mathrm{A}_{\text {sample }}\right) / \mathrm{A}_{\text {blank }}\right] \times 100 \%
$$

where $A_{\text {blank }}$ is the absorbance of the control reaction (containing all of the reagents except the test compound) and $\mathrm{A}_{\text {sample }}$ is the absorbance of the test samples. Exact concentration providing $50 \%$ inhibition $\left(\mathrm{IC}_{50}\right)$ was calculated from the graph plotted from the regression analysis as inhibition percentage against concentration of the medicinal materials. Gallic acid, ascorbic acid, and BHT were measured at the same procedure. Tests were carried out in triplicate. Results were expressed in milligram medicinal materials per milliliter $(\mathrm{mg} / \mathrm{mL})$.

\subsection{3. $\cdot$ OH Scavenging Activity Assay}

The scavenging ability of different extracts on hydroxide radical was measured in the $\mathrm{CuSO}_{4}-\mathrm{Phen}-\mathrm{Vc}-\mathrm{H}_{2} \mathrm{O}_{2}$ che- miluminescence (CL) system. The CL of hydroxyl radical formation was monitored under the described method [14] using a BPCL Ultra-weak luminescence analyzer (Institute of Biophysics, Academia Sinica, China). The extracts were dispersed in $1 \%$ Tween 20 and standards were dissolved in redistilled water, those solutions were diluted to fit the $\cdot \mathrm{OH}$ scavenging assay. The volume of the reaction was composed of $50 \mu \mathrm{L}$ of the sample solution, $50 \mu \mathrm{L}$ of $1.0 \mathrm{mmol} / \mathrm{L} \mathrm{CuSO}_{4}$ solution, $50 \mu \mathrm{L}$ of 1 $\mathrm{mmol} / \mathrm{L} 1,10$-phenanthroline solution, $700 \mu \mathrm{L}$ of 0.05 $\mathrm{mol} / \mathrm{L}$ borate buffer ( $\mathrm{pH} 9.0), 100 \mu \mathrm{L}$ of $1 \mathrm{mmol} / \mathrm{L}$ ascorbic acid solution, and $50 \mu \mathrm{L}$ of $1 \% \mathrm{H}_{2} \mathrm{O}_{2}$ solution. The reaction was initiated immediately after the injection of $\mathrm{H}_{2} \mathrm{O}_{2}$ solution, and kinetic curves were obtained at $2 \mathrm{~s}$ intervals over a period of $400 \mathrm{~s}$. Varying degrees of sudden drops of CL counts observed represent the different degrees of $\bullet \mathrm{OH}$ scavenging abilities. As the inhibiting percentage of CL counts had been calculated, comparison of the correlativity between the $\bullet \mathrm{OH}$ scavenging efficacy and the concentration of each sample is possible. The integrated area of the curve expressed the relative luminescent intensity. The scavenging activity was represented by the following formula:

$$
\begin{aligned}
\text { Inhibition } \% & =\frac{\left[\left(\mathrm{CL}_{\text {control }}-\mathrm{CL}_{0}\right)-\left(\mathrm{CL}_{\text {sample }}-\mathrm{CL}_{0}\right)\right]}{\left(\mathrm{CL}_{\text {control }}-\mathrm{CL}_{0}\right)} \\
& \times 100 \%
\end{aligned}
$$

where $\mathrm{CL}_{\text {control }}$ is the relative luminescent intensity of the control group, $\mathrm{CL}_{0}$ is the relative luminescent intensity of the background group, and $\mathrm{CL}_{\text {sample }}$ is the relative luminescent intensity of the experimental group. Exact concentration providing $50 \%$ inhibition $\left(\mathrm{IC}_{50}\right)$ was calculated from the graph plotted as scavenging percentage against concentration of medicinal materials. Gallic acid was also measured at the same procedure. Tests were carried out in triplicate. $\mathrm{IC}_{50}$ values were expressed in milligram medicinal materials per milliliter $(\mathrm{mg} / \mathrm{mL})$.

\subsection{4. $\mathrm{O}_{2}^{-}$Scavenging Activity Assay}

The $\mathrm{O}_{2}^{--}$scavenging activity of the selected herbs was determined by the nitrite reduction method [15]. The tested solutions were prepared in the $\cdot \mathrm{OH}$ scavenging assay and diluted to fit the $\mathrm{O}_{2}^{-}$scavenging assay. The reaction mixture contained $0.6 \mathrm{~mL} 1 \mathrm{mmol} / \mathrm{L}$ hypoxanthine, $0.3 \mathrm{~mL} 220 \mu \mathrm{mol} / \mathrm{L}$ hydroxylammonium-chloride, $1 \mathrm{~mL}$ buffer solution $(\mathrm{pH} 8.2$, the solution containing 15.6 $\mathrm{mmol} / \mathrm{L} \mathrm{Na}_{2} \mathrm{~B}_{4} \mathrm{O}_{7}$ and $20.8 \mathrm{mmol} / \mathrm{L} \mathrm{KH}_{2} \mathrm{PO}_{4}$ ), and $40 \mu \mathrm{L}$ $0.7 \mathrm{U} / \mathrm{mL}$ xanthine oxidase. The diluted solution of 1.0 $\mathrm{mL}$ was added to the reaction mixture and incubated for $30 \mathrm{~min}$ at $37^{\circ} \mathrm{C}$. Then $2.0 \mathrm{~mL} 1.73 \mathrm{mmol} / \mathrm{L}$ sulfanilic acid, which was dissolved in $1.36 \mathrm{mmol} / \mathrm{L}$ acetic acid, and $2.0 \mathrm{~mL}$ of $19.29 \mu \mathrm{mol} / \mathrm{L} \mathrm{N}$-1-naphthylethylenediamine were injected to the solution and shook. After standing at room temperature in the dark for $20 \mathrm{~min}$, the absorbance 
was measured at $550 \mathrm{~nm}$. A control solution was measured, in which sample was replaced by redistilled water. The scavenging rate was obtained according to the formula:

$$
\text { Scavenging rate }(\%)=\frac{A_{c}-A_{s}}{A_{c}-A_{b}} \times 100 \%
$$

where $A_{c}$ is the absorbance of the control solution, $A_{s}$ is the absorbance of the test sample, and $A_{b}$ represents the absorbance of the blank, in which xanthine oxidase was replaced by the buffer. Exact concentration providing 50 $\%$ inhibition $\left(\mathrm{IC}_{50}\right)$ was calculated from the graph plotted from the regression analysis as inhibition percentage against the concentration. The results were expressed in milligram raw materials per milliliter $(\mathrm{mg} / \mathrm{mL})$. Gallic acid and ascorbic acid were measured at the same procedure. Experiments were carried out in triplicate.

\subsection{Data Analysis}

Data were processed using origin 6.1 software (Microcal Software, Inc., Northampton, MA, USA). The regression equations and correlation coefficients were fitted by the least-squares method. All experiments were repeated at least three times. The results were expressed as means \pm SD. Standard differences were considered significant at $P<0.05$.

\section{Results and Discussions}

\subsection{Selection of Extraction Solvents}

In order to select the best solvent for extraction of those medicinal materials, four different percentages of ethanol $(10 \%, 3 \%, 60 \%$ and $95 \% \mathrm{v} / \mathrm{v})$ were used in the extraction of Folium artemistae argyi, Rhizoma rhodiolae crenulatae, and Cortex eucommiae respectively. The extraction solvent of $60 \%$ ethanol, indicated by the TPC values (Table 2), was found to give the highest extraction efficiency for the selected three herbs, while $95 \%$ ethanol had the lowest extraction efficiency. Consequently, $60 \%$ ethanol was chosen as the extraction solvent for the subsequent antioxidant assays. The average extraction efficiency of $60 \%$ ethanol was determined by multiple extraction experiments and was found in range from $95 \%$ to $97 \%$ after the first and the second extraction depending on the selected medicinal materials (Table 3). Therefore, the selected medicinal materials were extracted twice using $60 \%$ ethanol under refluxing for further investigations respectively.

\subsection{Total Phenolic Contents of 50 Medicinal Materials}

There was a wide range of the total phenolic contents among the selected medicinal materials. As shown in Ta- ble 1, the TPC values, determined by the Folin-Ciocalteau method, varied from 2 to $185 \mathrm{mg} \mathrm{GAE} / \mathrm{g}$ (average $39.9 \mathrm{mg} \mathrm{GAE} / \mathrm{g}$ ) depending on the biological origin of the plant. It is well known that plant polyphenols are widely distributed in the plant kingdom and sometimes in surprisingly high concentrations [16,17]. According to the results, there are 7 medicinal materials with the lowest total phenolics concentration $(<10 \mathrm{mg}$ GAE/g), including Semen nelumbinis $<$ Rhizoma atractylodis macrocephalae $<$ Flos magnolia officinalis $<$ Herba portulacae < Semen ginkgo < Folium mori < Radix dipsaci. Five herbs had total phenolics concentrations $>90 \mathrm{mg}$ GAE/g: Rhizoma rhodiolae crenulatae > Herba cirsii japonici $>$ Rhizoma sanguisorbae $>$ Radix rubiae $>$ Radix et rhizoma rhei. The highest total phenolics content ( $>150 \mathrm{mg} \mathrm{GAE} / \mathrm{g}$ ) was found in Rhizoma rhodiolae crenulatae, the roots collected from Rhodiola crenulata (Hook. f. et. Thoms.) H. Ohba. According to the literature [11], various phenolic compounds have different responses in TPC assay. The molar response of this method is roughly proportional to the number of phenolic hydroxyl groups in a given substrate, whereas the reducing capacity is enhanced when two phenolic hydroxyl groups are oriented ortho or para [18]. Since these structural features of phenolic compounds are also responsible for antioxidant activity, measurements of phenols in food or medicinal materials may be related to their antioxidant properties.

\subsection{Antioxidant Capacity}

\subsubsection{FRAP of 50 Medicinal Materials}

As shown in Table 1, the total antioxidant capacities (FRAP) are different from each other between the selected 50 medicinal materials. The FRAP values varied from 2 to 134 (the mean was calculated as 25.6) $\mathrm{mg}$ $\mathrm{GAE} / \mathrm{g}$ of the dried material weight. In FRAP assay, the

Table 2. Extraction efficiencies of Folium artemistae argyi, Rhizoma rhodiolae crenulatae, and Cortex eucommiae.

\begin{tabular}{cccc}
\hline Species & Extraction & $\begin{array}{c}\text { Average TPC } \\
\text { (mg GAE/g dried } \\
\text { sample) }\end{array}$ & $\begin{array}{c}\text { Average } \\
\text { extraction } \\
\text { efficiencies (\%) }\end{array}$ \\
\hline $\begin{array}{c}\text { Folium } \\
\text { artemistae } \\
\text { argyi }\end{array}$ & 1st & $30.09 \pm 0.67$ & $85.38 \pm 1.83$ \\
$\begin{array}{c}\text { Rhizoma } \\
\text { rhodiolae } \\
\text { crenulatae }\end{array}$ & 3rd & $3.61 \pm 0.16$ & $10.24 \pm 0.46$ \\
$\begin{array}{c}\text { Cortex } \\
\text { eucommiae }\end{array}$ & 3rd & $134 \pm 0.09$ & $4.37 \pm 0.28$ \\
& 2nd & $33.04 \pm 1.52$ & $17.76 \pm 0.83$ \\
& 3rd & $1.29 \pm 0.08$ & $3.20 \pm 0.94 \pm 0.45$ \\
\hline
\end{tabular}

${ }^{\mathrm{a}}$ The average of TPC and extraction efficiencies were based on triplicates from a single batch; the results are means $\pm \operatorname{SD}(n=3)$. 
Table 3. The total phenolic content (TPC) values and the in vitro antioxidant activities of fifty traditional Chinese medicines ${ }^{\mathrm{a}}$.

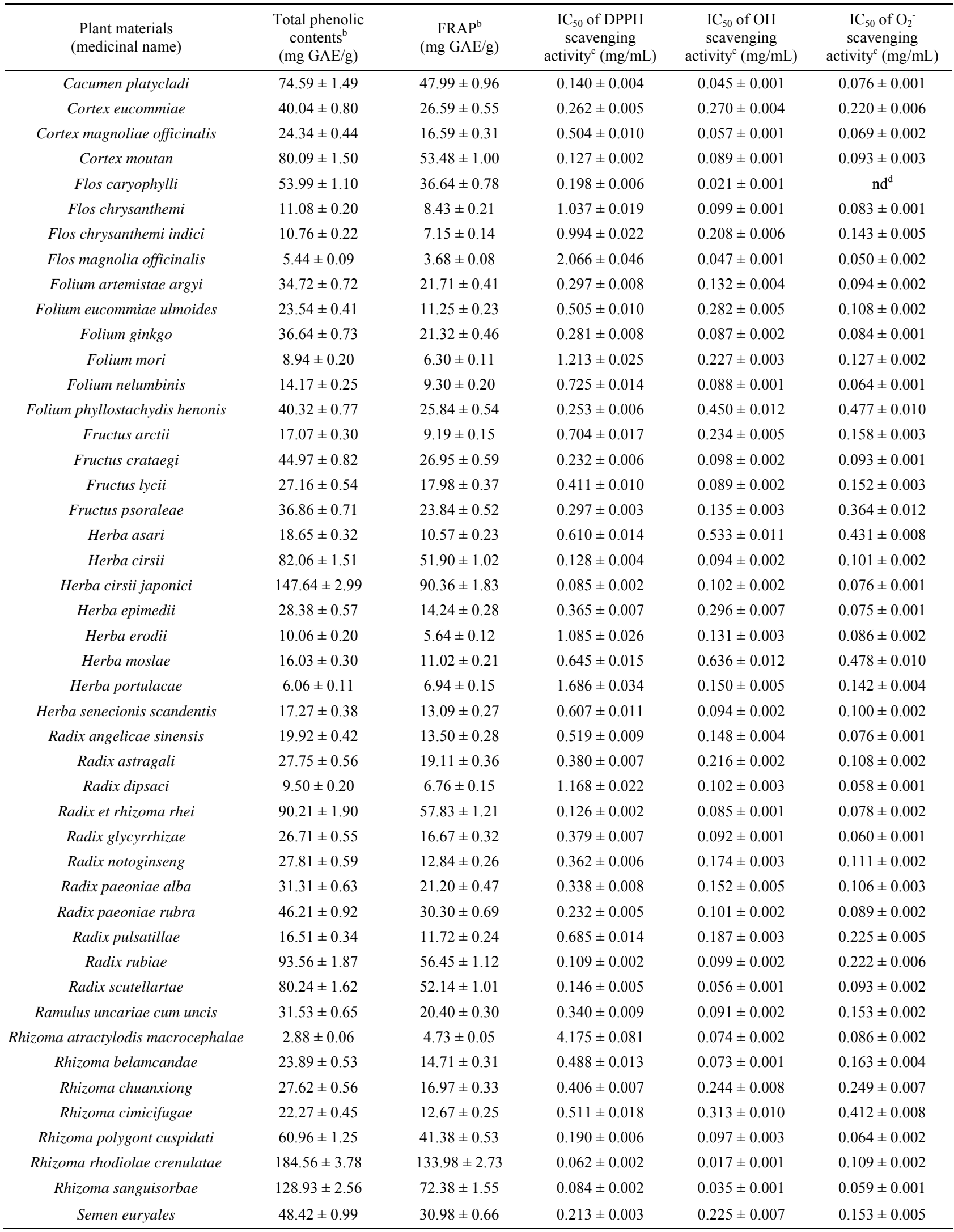


Continued

\begin{tabular}{cccccc}
\hline Semen ginkgo & $7.81 \pm 0.20$ & $4.96 \pm 0.07$ & $1.569 \pm 0.031$ & $0.217 \pm 0.003$ & $0.681 \pm 0.009$ \\
Semen nelumbinis & $2.20 \pm 0.05$ & $2.34 \pm 0.05$ & $5.477 \pm 0.111$ & $0.303 \pm 0.009$ & $0.125 \pm 0.003$ \\
Spica prunellae & $13.86 \pm 0.24$ & $9.17 \pm 0.16$ & $0.828 \pm 0.018$ & $0.216 \pm 0.006$ & $0.516 \pm 0.015$ \\
Thallus eckloniae & $59.97 \pm 1.22$ & $38.75 \pm 0.79$ & $0.175 \pm 0.005$ & $0.175 \pm 0.004$ & $0.151 \pm 0.006$ \\
Gallic acid & $-{ }^{-}$ & - & $0.0153 \pm 0.0004$ & $0.0123 \pm 0.0004$ & $0.101 \pm 0.003$ \\
Ascorbic acid & - & $510 \pm 7$ & $0.0042 \pm 0.0001$ & - & $0.0248 \pm 0.0006$ \\
BHT $^{\mathrm{e}}$ & - & - & $0.0191 \pm 0.0003$ & - & - \\
\hline
\end{tabular}

${ }^{\mathrm{a}}$ Results were means $\pm \mathrm{SD}(\mathrm{n}=3) ;{ }^{\mathrm{b}}$ Total phenolic contents were expressed in gallic acid equivalent of the dried medicinal materials; ${ }^{\mathrm{c}} \mathrm{IC} \mathrm{C}_{50}$ was defined as the concentration sufficient to obtain $50 \%$ scavenging activity; ${ }^{\mathrm{d}}$ The linear relation could not be constructed; ${ }^{\mathrm{e}}$ BHT represents butylated hydroxytoluene; ${ }^{\mathrm{f}}$ Not detected.

antioxidant activity was based on the ability of the antioxidant components in the samples to reduce $\mathrm{Fe}^{3+}$ to $\mathrm{Fe}^{2+}$ in a redox-linked colourimetric reaction that involves single electron transfer [12]. According to their reducing ability/antioxidant power (FRAP) values, 50 medicinal plants can be divided into five groups: a) very low FRAP ( $<5 \mathrm{mg} \mathrm{GAE} / \mathrm{g}$ ), $\mathrm{n}=4$; b) low FRAP (5 - $30 \mathrm{mg} \mathrm{GAE} / \mathrm{g}$ ), $\mathrm{n}=32$; c) good FRAP (30 - $50 \mathrm{mg}$ GAE/g), $\mathrm{n}=6$; d) high FRAP (50 - $100 \mathrm{mg} \mathrm{GAE} / \mathrm{g}), \mathrm{n}=7$; and e) very high FRAP $(>100 \mathrm{mg}$ GAE/g) $\mathrm{n}=1$. On the basis of the FRAP values of the selected chemicals, the ratio of the slope of the linear curve of ascorbic acid to that of $\mathrm{FeSO}_{4} \cdot 7 \mathrm{H}_{2} \mathrm{O}$ was 1.96 , and the ratio of gallic acid to $\mathrm{FeSO}_{4} \cdot 7 \mathrm{H}_{2} \mathrm{O}$ was 4.02 . Gallic acid, bearing a pyrogallol moiety, exhibited more potent activity than ascorbic acid (gallic acid vs asorbic acid = 2.05:1). The significant linear correlation (coefficient " $r$ " $=0.9918$, and twotailed "P"-value $<0.0001$ ) was confirmed between TPC values and their related FRAP values of the selected medicinal materials (Figure 1).

There are many methods to determine the total antioxidant capacity [19]. These in vitro and in vivo methods differ in terms of their assay principles and experimental conditions. Consequently, antioxidant components may individually have varying contributions to the total antioxidant capability in different methods. Because FRAP assay is quick and simple to perform, and the reaction is reproducible and linearly related to the molar concentration of the antioxidant(s) [20], the FRAP assay was applied in the determination of the total antioxidant capacity of those herbs. This method was initially developed to assay plasma antioxidant capacity, and popularly used to measure the antioxidant capacity from a wide range of biological samples in recent years, including teas, vegetables, fruits, wines, plants, and animal tissues [21,22]. In sharp contrast to the medicinal plants with high or very high FRAP values, the positive properties of the medicinal plants with very low FRAP are unlikely related to their antioxidant capacity. As a result, eight medicinal materials, namely Rhizoma rhodiolae crenulatae, Herba cirsii japonici, Rhizoma sanguisorbae, Radix et rhizoma rhei, Radix rubiae, Cortex moutan, Radix scutellartae, and Herba cirsii, have the highest FRAP values among those selected herbs.

\subsubsection{DPPH Radical Scavenging Activity}

DPPH assay was applied to test the ability of the antioxidative compounds as well as different plant extracts functioning as proton radical scavengers or hydrogen donors [23]. $\mathrm{IC}_{50}$ values of $\mathrm{DPPH}$ radicals scavenging activity were in the range of $0.06-5.50 \mathrm{mg} / \mathrm{mL}$ according to the results listed in Table 1. A negative correlation was found between the TPCs and $\mathrm{IC}_{50}$ values, indicating that the materials or the extracts at high TPC levels would have low $\mathrm{IC}_{50}$ values but strong potency to scavenge DPPH radicals. Among 50 selected materials, 12 medicinal materials with lowest $\mathrm{IC}_{50}$ values $(<0.2 \mathrm{mg} / \mathrm{mL})$ were Rhizoma rhodiolae crenulatae < Rhizoma sanguisorbae < Herba cirsii japonici < Radix rubiae < Radix et rhizoma rhei $<$ Cortex moutan $<$ Herba cirsii $<$ Cacumen platycladi $<$ Radix scutellartae $<$ Thallus eckloniae $<$ Rhizoma polygont cuspidati < Flos caryophylli. Gallic acid and BHT, determined with the $\mathrm{IC}_{50}$ values of $15.3 \mu \mathrm{g} / \mathrm{mL}$ and $19.6 \mu \mathrm{g} / \mathrm{mL}$ respectively, were used as the positive control in DPPH assay. The correlation was investigated between the concentration and the antioxidant capacity at different concentrations of individual medicinal materials. The results indicated that the selected herbs have liner relation between the DPPH radicals scavenging percentages and the TPC concentrations. It could be concluded that the DPPH radicals scavenging nature of those materials might depend on their total phenolics tentatively. As a result, a parabola regressive model could be built from those data. In other words, the reciprocals of the TPCs values were linear to the $\mathrm{IC}_{50}$ values (coefficient " $r$ " $=0.9985$, and two-tailed "P"-value $<0.0001$ ) (Figure 2).

\subsection{3. $\bullet \mathrm{OH}$ and $\mathrm{O}_{2}^{-}$Scavenging Activities}

To evaluate the ROS scavenging properties of those medicinal materials, we have used two different reactive oxygen species (ROS): the hydroxyl radical and superoxide anion radical. $\bullet \mathrm{OH}$ was produced and monitored by the $\mathrm{CuSO}_{4}$-Phen- $\mathrm{Vc}-\mathrm{H}_{2} \mathrm{O}_{2}$ chemiluminescence system, 


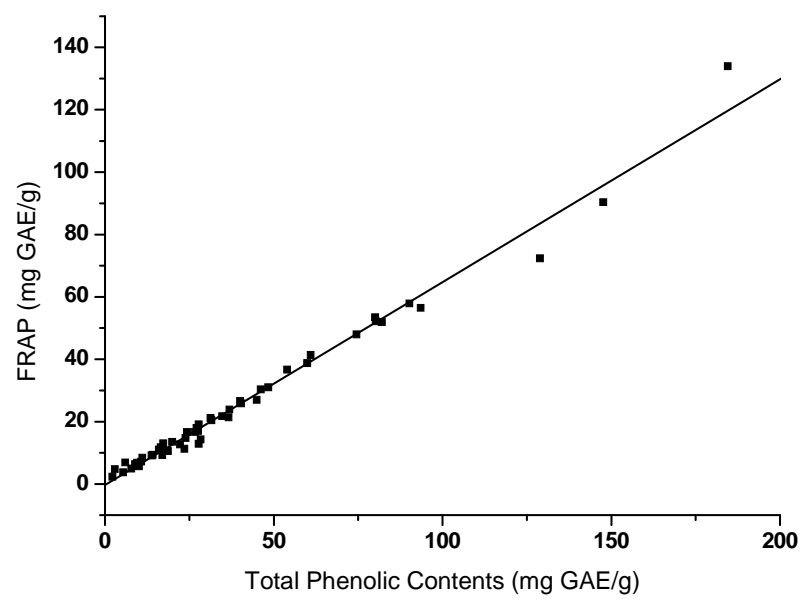

Figure 1. Linear correlation between the amount of total phenolics and the total antioxidant capacity (FRAP), $y=$ $0.6509 x-0.3783$. Correlation coefficient " $r$ " $=0.9918$. The two-tailed $P$ value is $<\mathbf{0 . 0 0 0 1}$, considered extremely significant.

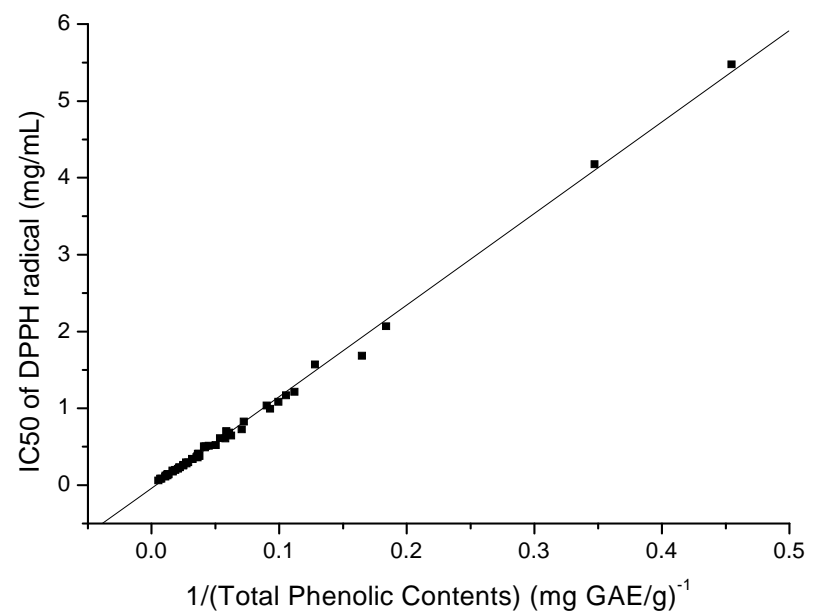

Figure 2. Linear correlation between the reciprocals of the total phenolic content and $\mathrm{IC}_{50}$ values of DPPH scavenging activity, $y=11.9209 x-0.0406$. Correlation coefficient " $r$ " = 0.9985. The two-tailed $P$ value is $<0.0001$, considered extremely significant.

whereas $\mathrm{O}_{2}^{-}$was generated by the hypoxanthinexanthine oxidase system and detected by UV-Vis spectrophotometry. The results of the ROS scavenging capacities, in the form of $\mathrm{IC}_{50}$ values of those herbs, were presented in Table 1. The $\mathrm{IC}_{50}$ values of $\bullet \mathrm{OH}$ and $\mathrm{O}_{2}^{--}$varied in the ranges of $0.017-0.636 \mathrm{mg} / \mathrm{mL}$ and 0.050 $0.681 \mathrm{mg} / \mathrm{mL}$ respectively. In the superoxide anion radical assay, only Flos caryophylli did not exhibit correlation between the free radicals scavenging percentage and the concentration. Comparison of the ROS scavenging characteristics of those medicinal materials, Herba moslae has the highest potency to scavenge the hydroxyl radicals, whereas Semen ginkgo is the highest in the superoxide anion radical assay. According to the results, there was a weak linear relation between $\mathrm{IC}_{50}$ values of the hydroxyl radical and the superoxide anion radical scavenging activities (coefficient " $r$ " $=0.6442$, and two-tailed "P"-value $<0.0001$ ). The individual extracts, which could scavenge the hydroxyl radicals, can not necessarily eliminate the superoxide anion radicals. As a result, traditional Chinese herbs have specific ROS scavenging properties respectively, which can be applied in the explanation of the rules of compatibility of medicines in the traditional Chinese medicine.

In the ROS scavenging experiments, there was no linear response between the total phenolic contents and the free radicals scavenging activities, other factors should be considered in the evaluation of the ROS scavenging capacities. There were several methods to screen the ROS scavengers, different methods could give varied results for the unstable characteristics of the ROS in chemical or biochemical systems. The total phenolics content in the extracts could be correlated linearly with the oxygen depletion, but not with the ROS scavenging effect by different methods using ESR spin trapping and electrochemical measurement [24]. Other scientists also have not found linear response between the total phenolics and the ROS scavenging capacities [8]. The difference between the sterical structures of antioxidants or the free radicals played a more important role in the abilities to scavenge different types of free radicals [25], which could be applied in the explanation of the antioxidant variations between the $\mathrm{DPPH} \cdot, \cdot \mathrm{OH}$, and $\mathrm{O}_{2}^{-}$scavenging activities. In DPPH assay, the $60 \%$ ethanol extracts showed higher scavenging activity than the $95 \%$ ethanol extracts. The similar conclusion could be drawn from the results of the extracts by different polar solvents in $\cdot \mathrm{OH}$ and $\mathrm{O}_{2}^{-}$assays. It suggests that more-polar components presented in extracts have contributed towards the increased ROS scavenging activities. Although there was no direct evidence in this study, the antioxidant activities of $60 \%$ ethanol extracts could be related to the presence of phenolic compounds, peptides, saccharides, and other polar compounds because they contain hydroxyl moiety $[26,27]$.

\subsubsection{Comparison of Antioxidant Activities of 50 Medicinal Materials}

Influenced by several biofactors, such as the ROS and other free radicals occurrence, the redox status in human body, and the bioavailability of the phytochemicals, the traditional Chinese herbs would act as more complicated roles in the life processes than the chemical or biochemical systems in vitro. According to the classification of their medicinal usages in the traditional Chinese medicine, 18 of those herbs, including 11 stanchers, were traditionally used as the haematic. Those haematic drugs, especially the stanchers, have highest TPC values and 
strongest antioxidant capacities in comparison with other herbs. The next is the heat-clearing drugs, totally 10 herbs are ranged to this class. According to the results, those heat-clearing drugs owned higher total phenolic contents and moderate antioxidant activities. The other medicinal materials, traditionally defined as the tonic, the diaphoretic and damp-resolving, have quite low TPC values and low antioxidant activities. On the other hand, the diseases are usually treated by complex prescriptions using the drug matching principles in traditional Chinese medicine. So, it would be of great importance to investigate the antioxidant characteristics of the traditional Chinese medicinal materials using the different antioxidant screening systems.

The total antioxidant (FRAP) and $\mathrm{DPPH} \bullet, \cdot \mathrm{OH}$ and $\mathrm{O}_{2}^{--}$scavenging activities have different mechanisms in the antioxidant effects, so the herbs with the highest capacities were chose as the potential antioxidants. Four traditional Chinese herbs, namely Cacumen platycladi, Radix et rhizoma rhei, Rhizoma rhodiolae crenulatae and Rhizoma sanguisorbae, have antioxidant potency in comparison with some well known natural and synthetic antioxidants. Contrarily, Folium mori, Fructus arctii, Semen ginkgo, Semen nelumbinis and Spica prunellae were the weak antioxidants correspondingly. It has been revealed that various phenolic antioxidants, such as flavonoids, tannins, coumarins, xanthones, and procyanidins, can scavenge free radicals dose-dependently [28], thus they are viewed as promising therapeutic drugs for the free radical related disorders or illnesses. There are more than 4000 naturally occurring flavonoids described in the literature [29], including chalcones, flavonones, flavones, biflavonoids, dihydroflavonols, anthrocyanidins, and flavonols. Other polar natural products, such as proteins, saccharides, etc., also have the antioxidant capacities, but as a rule, phenolic compounds were applied in the evaluation of the correlation between the results of the antioxidant capacities and the botanic materials [26,27]. Therefore, the antioxidant activities of plant original medicinal materials are dependent on the chemical type of antioxidant compounds, the polarity of the extracting solvent, and the test systems or the substrates to be protected.

Interestingly, many complex prescriptions can be assembled from the selected 50 herbs according to the traditional Chinese medicine. Among those prescriptions, the herbs from at least two types are discriminated as monarch, minister, assistant and guide by the roles of their actions in the diseases treatment. The composition of abundant substances in the complex prescription will provide more complicated and synergistic antioxidant effects in the human body than the individual herbs.

\section{Conclusion}

In conclusion, our results further support the point of view that some medicinal materials are promising sources of natural antioxidants. Among 50 selected traditional Chinese herbs, the total phenolic content and the antioxidant capacity differed significantly. There were significant linear correlations between the total phenolic concentration and the values of FRAP or DPPH radicals scavenging percentage. We also have found that three stanchers, namely Cacumen platycladi, Rhizoma Rhodiolae crenulatae, Rhizoma sanguisorbae, and one cathartic, that is Radix et rhizoma rhei, have significant ferric reducing power and free radicals scavenging activities. Those traditional Chinese medicines have been certified with low profile of side effects and toxicities for thousands of years. Several herbs, popularly used in the traditional Chinese medicine, have already been on schedule to be investigated for their phytochemistry and their medicinal applications.

\section{Acknowledgements}

We gratefully acknowledge Shanghai Nanotechnology Promotion Center (Grant No. 0552nm018) for the financial support.

\section{REFERENCES}

[1] B. Halliwell and M. C. G. John, "Free Radicals in Biology and Medicine," 2 Edition, Oxford University Press, Oxford, 1989, pp. 299-508.

[2] N. Ito, S. Fukushima and H. Tsuda, "Carcinogenicity and Modification of the Carcinogenic Response by BHA, BHT, and Other Antioxidants," Critical Reviews in Toxicology, Vol. 15, No. 2, 1985, pp. 109-150.

http://dx.doi.org/10.3109/10408448509029322

[3] R. G. Cutler, "Antioxidants and Aging," The American Journal of Clinical Nutrition, Vol. 53, No. 1, 1991, pp. 373S-379S.

[4] X. M. Hu, "Chinese Materia Medica (in Chinese)," Vol. 1, Shanghai Science and Technology Press, Shanghai, 1999, pp. 1-252.

[5] Y. Cai, et al., "Antioxidant Activity and Phenolic Compounds of 112 Traditional Chinese Medicinal Plants Associated with Anticancer," Life Sciences, Vol. 74, No. 17, 2004, pp. 2157-2184.

http://dx.doi.org/10.1016/j.lfs.2003.09.047

[6] P. Scartezzini and E. Speroni, "Review on Some Plants of Indian Traditional Medicine with Antioxidant Activity," Journal of Ethnopharmacology, Vol. 71, No. 1-2, 2000, pp. 23-43. http://dx.doi.org/10.1016/S0378-8741(00)00213-0

[7] Y. Z. Cai, et al., "Structure-Radical Scavenging Activity Relationships of Phenolic Compounds from Traditional Chinese Medicinal Plants," Life Sciences, Vol. 78, No. 25, 2006, pp. 2872-2888. http://dx.doi.org/10.1016/j.lfs.2005.11.004

[8] B. Ou, et al., "When the East Meets West: The Relationship between Yin-Yang and Antioxidation-Oxidation," 
The FASEB Journal: Official Publication of the Federation of American Societies for Experimental Biology, Vol. 17, No. 2, 2003, pp. 127-129.

[9] Y. T. Szeto and I. F. Benzie, "Is the Yin-Yang Nature of Chinese Herbal Medicine Equivalent to AntioxidationOxidation?" Journal of Ethnopharmacology, Vol. 108, No. 3, 2006, pp. 361-366. http://dx.doi.org/10.1016/i.jep.2006.05.033

[10] D. Wormuth, et al., "Redox Regulation and Antioxidative Defence in Arabidopsis Leaves Viewed from a Systems Biology Perspective," Journal of Biotechnology, Vol. 129, No. 2, 2007, pp. 229-248. http://dx.doi.org/10.1016/j.jbiotec.2006.12.006

[11] V. L. Singleton and J. A. Rossi, "Colorimetry of Total Phenolics with Phosphomolybdic-Phosphotungstic Acid Reagents," American Journal of Enology and Viticulture, Vol. 16, No. 3, 1965, pp. 144-158.

[12] I. F. F. Benzie and J. J. Strain, "The Ferric Reducing Ability of Plasma (FRAP) as a Measure of "Antioxidant Power': The FRAP Assay," Analytical Biochemistry, Vol. 239, No. 1, 1996, pp. 70-76. http://dx.doi.org/10.1006/abio.1996.0292

[13] T. Katsube, et al., "Screening for Antioxidant Activity in Edible Plant Products: Comparison of Low-Density Lipoprotein Oxidation Assay, DPPH Radical Scavenging Assay, and Folin-Ciocalteu Assay," Journal of Agricultural and Food Chemistry, Vol. 52, No. 8, 2004, pp. 2391-6. http://dx.doi.org/10.1021/jf035372g

[14] C. H. Tsai, et al., "Rapid and Specific Detection of Hydroxyl Radical Using an Ultraweak Chemiluminescence Analyzer and a Low-Level Chemiluminescence Emitter: Application to Hydroxyl Radical-Scavenging Ability of Aqueous Extracts of Food Constituents," Journal of Agricultural and Food Chemistry, Vol. 49, No. 5, 2001, pp. 2137-2141. http://dx.doi.org/10.1021/jf001071k

[15] Y. Oyangui, "Reealuative of Assay Methods and Establishment of Kit for Superoxide Dismutase Activity," Analytical Biochemistry, Vol. 142, No. 2, 1984, pp. 290-296. http://dx.doi.org/10.1016/0003-2697, No. 84)90467-6

[16] J. B. Harborne and C. A. Williams, "Anthocyanins and Other Flavonoids," Natural Product Reports, Vol. 18, No. 3, 2001, pp. 310-333. http://dx.doi.org/10.1039/b006257j

[17] J. B. Harborne and C. A. Williams, "Advances in Flavonoid Research Since 1992," Phytochemistry, Vol. 55, No. 6, 2000, pp. 481-504. http://dx.doi.org/10.1016/S0031-9422(00)00235-1

[18] E. N. Frankel, A. L. Waterhouse and P. L. Teissedre, "Principal Phytochemicals in Selected California Wines and Their Antioxidant Activity in Inhibiting Oxidation of Human Low-Density Lipoproteins," Journal of Agricul-

\footnotetext{
Abbreviations

FRAP: ferric reducing/antioxidant power

GAE: gallic acid equivalent

IC $_{50}$ : exact concentration providing $50 \%$ inhibition

BHT: butylated hydroxytoluene

DPPH•: the free radical of di(phenyl)-(2,4,6-trinitro-
}

tural and Food Chemistry, Vol. 43, No. 4, 1995, pp. 890894. http://dx.doi.org/10.1021/jf00052a008

[19] G. Bartosz, "Total Antioxidant Capacity," In: H. E. Spiegel, Ed., Advances in Clinical Chemistry, Vol. 37, 2003, Academic Press, New York, pp. 219-292.

[20] I. F. F. Benzie, W. Y. Chung and J. J. Strain, "Antioxidant (Reducing) Efficiency of Ascorbate in Plasma Is Not Affected by Concentration," The Journal of Nutritional Biochemistry, Vol. 10, No. 3, 1999, pp. 146-150. http://dx.doi.org/10.1016/S0955-2863(98)00084-9

[21] D. Modun, et al., "The Increase in Human Plasma Antioxidant Capacity after Red Wine Consumption Is Due to Both Plasma Urate and Wine Polyphenols," Atherosclerosis, 2007.

[22] V. Katalinic, et al., "Screening of 70 Medicinal Plant Extracts for Antioxidant Capacity and Total Phenols," Food Chemistry, Vol. 94, No. 4, 2006, pp. 550-557. http://dx.doi.org/10.1016/j.foodchem.2004.12.004

[23] T. Nomura, et al., "Proton-Donative Antioxidant Activity of Fucoxanthin with 1,1-Diphenyl-2-picrylhydrazyl (DPPH)," Biochemistry and Molecular Biology International, Vol. 42, No. 2, 1997, pp. 361-370.

[24] H. L. Madsen, et al., "Screening of Antioxidantive Activity of Spices. A Comparison between Assays Based on ESR Spin Trapping and Electrochemical Measurement of Oxygen Consumption," Food Chemistry, Vol. 57, No. 2, 1996, pp. 331-337. http://dx.doi.org/10.1016/0308-8146(95)00248-0

[25] Q. Guo, et al., "ESR and Cell Culture Studies on Free Radical-Scavenging and Antioxidant Activities of Isoflavonoids," Toxicology, Vol. 179, No. 1-2, 2002, pp. 171180. http://dx.doi.org/10.1016/S0300-483X(02)00241-X

[26] L. E. Netto, et al., "Reactive Cysteine in Proteins: Protein Folding, Antioxidant Defense, Redox Signaling and More. Comparative Biochemistry and Physiology," Toxicology \& Pharmacology: CBP, Vol. 146, No. 1-2, 2007, pp. 180193.

[27] A. Kardosová and E. Machová, “Antioxidant Activity of medicinal Plant Polysaccharides," Fitoterapia, Vol. 77, No. 5, 2006, pp. 367-373. http://dx.doi.org/10.1016/j.fitote.2006.05.001

[28] Y. C. Zhou and R. L. Zheng, "Phenolic Compounds and an Analog as Superoxide Anion Scavengers and Antioxidants," Biochemical Pharmacology, Vol. 42, No. 6, 1991, pp. 1177-1179. http://dx.doi.org/10.1016/0006-2952(91)90251-Y

[29] E. Grotewold, "The Science of Flavonoids," Springer Science \& Business Media, Inc., New York, 2006, pp. $1-47$.

phenyl)iminoazanium

ROS: reactive oxygen species

RNS: reactive nitrogen species

TPC: total phenolic content

CL: chemiluminescence 